\title{
Is it time to end lone-wolf medicine?
}

\section{Andrew Loblaw, MD}

Department of Radiation Oncology, Sunnybrook Health Sciences Centre, University of Toronto, Toronto, ON, Canada

Cite as: Can Urol Assoc J 2016;10(9-10):319-20. http://dx.doi.org/10.5489/cuaj.4163

\section{See related article on page 314 .}

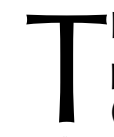

here is little uncertainty about what constitutes appropriate care of a patient with high-risk features (HRF) (positive margin, extracapsular extension, and/or seminal vesicle involvement) after radical prostatectomy (RP). There are three randomized, controlled trials (RCTs) showing improvements in local and biochemical control for the use of adjuvant postoperative radiation (aRT) $;^{1-3}$ the study with the longest followup (SWOG 8794) also showed an improvement in distant metastatic disease-free survival and overall survival (OS). ${ }^{2}$ With this strong evidentiary base, there are now a number of high-quality, evidence-based guidelines recommending that any patient with a high-risk feature be referred to a radiation oncologist for discussion about the pros/cons of aRT. These groups include Cancer Care Ontario (CCO), ${ }^{4}$ American Urological Association/ American Society for Radiation Oncology (AUA/ASTRO), ${ }^{5}$ The National Institute for Health and Care Excellence (NICE), ${ }^{6}$ and American Society of Clinical Oncology (ASCO). ${ }^{7}$ In my opinion, it is rare to have consistent recommendations framed in guidelines from different jurisdictions even when using the same evidentiary base.

Taggar et al demonstrate for us how often these recommendations are being followed and importantly, how patterns have changed over time. They describe the findings from a chart audit, population-based study of RP patients who were referred in Alberta during two time periods, 2005 and 2012, before and after the three RCTs were published.

It is notable that the proportion of patients receiving RP has dropped from $36.6 \%$ in 2005 to $24 \%$ in 2012. This downward trend mirrors Canadian Institute for Health Information $(\mathrm{CIHI})$ data across Canada. ${ }^{8}$ The authors suggest that fewer low-risk patients received RP, while there was a modest increase in the proportion treated with brachytherapy. Reassuringly, R1, seminal vesicle (SV) involvement, detectable prostate-specific antigen (PSA), and LN+ rates all dropped, suggesting better case selection, grade migration, better surgery, or combinations thereof.

While more patients (20.6\%) were referred in adjuvant setting (i.e., within six months of RP) in 2012 (vs. 12.9\% in 2005), this really represented a shift from the early salvage to adjuvant setting. In fact, overall referral rates dropped from $43.6 \%$ in 2005 to $27.9 \%$ in 2012 despite much lower median wait times (26.4 vs. 3.7 months, respectively) and more high-risk patients being operated on. Focusing on the patients who met guideline criteria (HRF and undetectable PSA), more patients were referred (30\% in 2012 vs. $24 \%$ in 2005), but still a large proportion (approximately 70\%) were not referred, as per guidelines.

The first question is why weren't more men referred? Certainly it takes time to disseminate information from trials and guidelines to the practicing populous. Jin et al have recently published a similar population-based study from Ontario showing similar trends - an increase in the number of patients referred to radiation oncology within six months of RP between 2003 and 2004 and 2011 and 2012, but the proportion referred tops out at $21.7 \%$, two years after the long-term results of SWOG 8794, where the OS advantage was reported. ${ }^{9}$ So it doesn't appear to be dissemination time that is the major factor. These authors could examine whether referral rates for academic vs. community urologists was different to test this hypothesis.

It could be that men don't want to travel long distances for a radiotherapy consultation and/or treatment. While a small proportion of men may choose to forego their only curative salvage option, the average age of men getting RP in the Alberta study was 62 years old, so I can't believe that distance alone explains the $70 \%$ non-compliance rate. Certainly in Ontario, where there are 14 radiotherapy (RT) centres (compared the Alberta's two RT centres), there's likely less driving required and yet even lower referral rates are seen $(22 \%)$. Each of these groups could do a subset analysis by driving distance to the closest RT centre to see how powerful a factor that is.

Some physicians and patients may perceive RT to be too toxic (despite the benefits). This shouldn't be a large 
deterrent to going to a consultation to talk about the pros/ cons, but some may believe if it's too toxic, why go to the appointment in the first place? The reality is, that postoperative radiation has a very low risk of serious toxicity; in the Wiegel trial, $1 \%$ and $0 \%$ of patients had Grade 3 bladder and bowel problems, respectively. ${ }^{3}$ This is despite patients receiving two-dimensional $R T$ in the Wiegel trial. $R T$ has improved greatly in target localization with image-guided techniques and my experience is that modern RT has even fewer side effects.

In my practice, many patients are given misleading information about the risk of second cancers after radiation, citing a $60 \%$ increased risk of getting a second cancer (hazard ratios of 1.6 for RP + RT v.s RP alone ${ }^{10}$ ). However, also reported in that same paper, but in my experience rarely mentioned to patients, is that the absolute increased risk of second cancer at 10 years is $1.5 \%$ and that on propensitybased multivariate analyses, this difference is not statistically significant. ${ }^{10}$ In my mind, even if there were a real but small increased second cancer risk, this is negligible compared to an absolute recurrence risk advantage of $20 \%$ and an $8 \%$ OS difference at 10 years for aRT. , $^{2,5}$

Lastly, many urologists I've spoken to believe that aRT over-treats patients who would never fail biochemically and that early salvage RT is equally effective. The chance of being recurrence-free at 15 years is about $30 \%$ when at least one high-risk feature is present without aRT, so to some extent that's true, but most will still benefit from aRT. ${ }^{2}$ With respect to the hypothesis that early salvage RT is as effective as adjuvant, Surveillance, Epidemiology, and End Result (SEER) data does show lower cause-specific mortality, bonerelated events, and salvage hormonal therapy use when early aRT ( $<4$ months) is used for pT3 disease (compared to 4-12 months postoperatively). ${ }^{11}$ Ultimately, this hypothesis needs to be confirmed in a properly designed and conducted clinical trial. The MRC RADICALS study (radiation timing arm) is addressing this question, but won't close until later this year and won't read out for at least five years after that. In the meantime, men should be counselled on the use of aRT if they have high-risk features postoperatively. Lone-wolf physicians who are convinced they know better or "don't believe" randomized data, I believe will increasingly have more pressure, fewer resources, and greater financial penalties assessed as our cancer system moves to higher-quality, patient-centred care. Let's end the era of lone-wolf medicine earlier rather than later for all our sakes.

Competing interests: The author reports no competing personal or financial interests.

\section{References}

1. Bolla $M$, van Poppel $H$, Collette $L$, et al. Postoperative radiotherapy after radical prostatectomy: A randomized, controlled trial (EORTC trial 22911). Lancet 2005;366:572-8. http://dx.doi. org/10.1016/S0140-6736(05)67101-2

2. Thompson $I M$, Tangen $C M$, Paradelo J, et al. Adjuvant radiotherapy for pathological T3NOMO prostate cancer significantly reduces risk of metastases and improves survival: Long-term followup of a randomized clinical trial. J Urol 2009;22:22. http://dx.doi.org/10.1016/i.juro.2008.11.032

3. Wiegel I, Bottke D, Steiner U, et al. Phase 3 postoperative adjuvant radiotherapy after radical prostatectomy compared with radical prostatectomy alone in pT3 prostate cancer with postoperative undetectable prostate-specific antigen: ARO 96-02/AUO AP 09/95. J Clin Oncol 2009;27:2924-30. http://dx.doi. org/10.1200/JC0.2008.18.9563

4. Morgan SC, Waldron TS, Eapen L, et al. Adjuvant radiotherapy following radical prostatectomy for pathologic T3 or margin-positive prostate cancer: A systematic review and meta-analysis. Radiother Oncol 2008;88:1-9. http://dx.doi.org/10.1016/i.radonc.2008.04.013

5. Thompson IM, Valicenti RK, Albertsen $P$, et al. Adjuvant and salvage radiotherapy after prostatectomy: AUA/ASTRO guideline. J Urol 2013;190:441-9. http://dx.doi.org/10.1016/i.juro.2013.05.032

6. National Institute for Heath and Care Excellence: Prostate Cancer: Diagnosis and Treatment, 2014. Avaiable at https://www.nice.org.uk/guidance/cg175?unlid. Accessed September 6, 2016.

7. Freedland SJ, Rumble RB, Finelli A, et al. Adjuvant and salvage radiotherapy after prostatectomy: American Society of Clinical Oncology clinical practice guideline endorsement. J Clin Oncol 2014;32:3892-8. http://dx.doi.org/10.1200/JC0.2014.58.8525

8. Canadian Institute for Health Information: The Delivery of Radical Prostatectomy to Treat Men With Prostate Cancer, 2014. Available at https://secure.cihi.ca/free_products/ProstateSurgeryinCanada_EN_web.pdf. Accessed September 6, 2016.

9. Jin CJ, Brundage MD, Cook EF, et al. Quality of radiation therapy referral and utilisation post-prostatectomy: A population-based study of time trends. Clin Oncol 2016 [Epub ahead of print]. http://dx.doi. org/10.1016/i.clon.2016.07.006

10. Wallis $\mathrm{CJ}$, Mahar AL, Choo R, et al. Second malignancies after radiotherapy for prostate cancer: Systematic review and meta-analysis. BMJ 2016;352:1851. http://dx.doi.org/10.1136/bmi.i851

11. Kowalczyk KJ, Gu X, Nguyen PL, et al. Optimal timing of early versus delayed adjuvant radiotherapy following radical prostatectomy for locally advanced prostate cancer. Urol Oncol 2014;32:303-8. http://dx.doi.org/10.1016/i.urolonc.2013.09.004

Correspondence: Dr. Andrew Loblaw, Sunnybrook Health Sciences Centre, Toronto, ON, Canada; andrew.loblaw@sunnybrook.ca 